superselective segmental renal artery catheterization and embolization as therapeutic options for such cases. Patients and Methods: Over the last 8 years, 28 patients with severe renal hemorrhage were admitted for evaluation and possible further treatment. Twenty of them had a history of previous biopsy ( 6 of them one of a transplanted kidney), 1 patient had a recent percutaneous nephrostomy, 4 patients presented with renal mass ruptures (2 patients renal cell carcinoma, 1 patient angiomyolipoma, 1 patient hemorrhagic cysts), 1 patient had rupture of a renal aneurysm during delivery, 1 patient suffered bleeding after partial nephrectomy, and 1 patient was hospitalized after a car accident. They all presented with clinical signs of hemodynamic instability. Angiographic investigation of the kidneys preceded further intervention in all cases. 26 out of the 28 patients underwent superselective embolization of the specific bleeding vessel with the use of microcoils and/or Gelfoam particles. Results: All patients treated by superselective segmental renal artery embolization had a successful outcome, including a steady renal function and a stable clinical course. No complications occurred.

Conclusion: Superselective segmental renal artery catheterization and embolization is a safe and efficient method for the treatment of patients with severe renal hemorrhage, preserving healthy renal parenchyma and renal function.

\title{
Editorial Comment
}

Increasingly, there is wide acceptance and support in the literature for the nonoperative management of nearly all blunt renal trauma, except for the potentially life-threatening injuries that are AAST Grade V. An aggressive approach at nonoperative management, inherently accepts an increased complication rate of delayed bleed or urinary leak. However, such complications can be effectively managed endoscopically or endo-vascularly. Delayed renal bleeding can occur up to several weeks after initial injury, although the period of greatest risk is within the first two to three weeks after injury. Clot lysis and hematoma liquefaction begins around day 5 to 7 and continues for another 2 weeks or so. It is during this time that renal bleeding is most likely. The kidney can bleed either into the collecting system, into the perirenal space, or as an arteriovenous fistula. Overall, delayed bleeding after trauma is rare, effecting less then one percent of all renal injuries. Penetrating injuries managed conservatively, in particular stab wounds, are especially prone to delayed bleeding, occurring in up to $18 \%$ of cases. Conservatively managed large devascularized renal segments with an associated bowel injury are also prone to delayed hemorrhage.

Dr. Steven B. Brandes Associate Professor, Division of Urologic Surgery Washington University in St. Louis

St. Louis, Missouri, USA

\section{PATHOLOGY}

\section{What's New in Prostate Cancer Disease Assessment in 2006?}

Epstein JI

Departments of Disease, Urology, and Oncology, The Johns Hopkins Hospital, Baltimore, Maryland, USA

Curr Opin Urol. 2006; 16: 146-51

Purpose of Review: Issues relating to the disease are critical in the diagnosis, management, and prognostication of prostate cancer. 
Recent Findings: New data have emerged regarding the disease of prostate cancer and its precursors. The diagnosis of prostate cancer on needle biopsy has been refined because of the recent discovery of alphamethylacyl-CoA racemase, which preferentially labels adenocarcinoma of the prostate. Modifications and additions to the Gleason grading system were published based on a consensus conference of urological pathologists. Various models have been proposed using Gleason score, clinical findings, as well as measurements of tumor volume on needle biopsy to enhance the prediction in men undergoing radical prostatectomy and to predict "insignificance". Several studies have confirmed that certain findings in radical prostatectomy are adverse, yet conflicting studies were published as to the independent prognosis of tumor volume. The risk of cancer following a diagnosis of high-grade prostatic intraepithelial neoplasia on needle biopsy has decreased to the point at which this author does not recommend a routine repeat needle biopsy within the first year following the diagnosis of high-grade prostatic intraepithelial neoplasia.

Summary: This review summarizes developments over the last year in the disease of prostate cancer and its precursors.

\section{Editorial Comment}

Alpha-methylacyl coenzyme A racemase (AMACR) is an enzyme first purified and characterized by investigators studying lipid metabolism. Although the role of this enzyme is still unknown, the application of AMACR immunohistochemistry in prostate pathology has been increased sharply in the last few years. AMACR preferentially labels adenocarcinoma of the prostate. In cases that the basal cells are patchy or discontinuous this labeling may be very useful for the differential diagnosis. Unfortunately, AMACR does not stain exclusively neoplastic acini. Benign acini may also show positivity, therefore, this stain should be used with caution (1).

Due to the widespread use of extended needle prostatic biopsies, Epstein does not recommend a routine repeat needle biopsy within the first year following the diagnosis of high-grade prostatic intraepithelial neoplasia. This recommendation is based on studies showing a substantially decreasing in subsequent cancer detection if high-grade prostatic intraepithelial neoplasia is seen in extended biopsies (2).

An important contribution for the improvement of the Gleason grading system was given by a Consensus Conference sponsored by the International Society of Urological Pathology (ISUP) during the USCAP (United States and Canadian Academy of Pathology) meeting in San Antonio, Texas, in 2005 (3).

There are several arguments favoring a need for a consensus on Gleason grading: 1)- In the 1960s, there was no screening for prostate cancer other than by digital rectal examination, 2)- The use of 18-gauge thin biopsy needles and the concept of sextant needle biopsies to more extensively sample the prostate were not developed until the 1980s, 3)- Tertiary patterns were not addressed within the original Gleason system, 4)- The Gleason system predated the use of immunohistochemistry (it is likely that many of Gleason's original $1+1=$ 2 adenocarcinomas would today be regarded as adenosis, 5)- The original Gleason grading system was not applied to newly described variants of adenocarcinoma of the prostate; and, 6)- The Gleason system varies considerably in contemporary surgical pathology practice and has led to several recent attempts to achieve consensus on Gleason grading.

A recent study in our Institution (4) showed that the highest impact of the consensus recommendations on a series of cases graded according to the standard Gleason system was seen on the secondary pattern, which had the lowest percentage of concordance. It reflected in a change toward a higher Gleason grading group in 46/ $172(26.74 \%)$ of the cases. A further study showed that these 46 patients had significantly a higher preoperative PSA, had more extensive tumors in the surgical specimen, had higher frequency of positive surgical margins and pathologic stage, and a strong tendency for shorter time to biochemical (PSA) progression-free survival following radical prostatectomy. The recommendations of the ISUP are a valuable refinement for the standard Gleason grading system and should be used by pathologists in their routine practice. 


\section{References}

1. Jiang Z, Fanger GR, Woda BA, Banner BF, Algate P, Dresser K, et al.: Expression of alpha-methylacyl-CoA racemase (P504s) in various malignant neoplasms and normal tissues: a study of 761 cases. Hum Pathol. 2003; 34: 792-6.

2. Herawi M, Kahane H, Cavallo C, Epstein JI: Risk of prostate cancer on first re-biopsy within 1 year following a diagnosis of high grade prostatic intraepithelial neoplasia is related to the number of cores sampled. J Urol. 2006; 175 : 121-4.

3. Epstein JI, Allsbrook WC Jr, Amin MB, Egevad LL; ISUP Grading Committee: The 2005 International Society of Urological Pathology (ISUP) Consensus Conference on Gleason Grading of Prostatic Carcinoma. Am J Surg Pathol. 2005; 29: 1228-42.

4. Guimaraes MS, Billis A, Quintal MM, Magna LA, Ferreira U: The impact of the 2005 International Society of Urological Pathology (ISUP) Consensus Conference on standard Gleason grading of prostatic carcinoma. Mod Pathol Suppl. 2006; 1: 139A.

Dr. Athanase Billis

Full-Professor of Pathology

State University of Campinas, Unicamp

Campinas, Sao Paulo, Brazil

\section{Prostate Basal Cell Lesions Can Be Negative for Basal Cell Keratins: A Diagnostic Pitfall}

Zhou M, Magi-Galluzzi C, Epstein JI

Department of Anatomic Pathology, The Cleveland Clinic Foundation, Cleveland, Ohio, USA

Anal Quant Cytol Histol. 2006; 28: 125-9

Background: Prostate basal cell lesions can have architectural and cytologic atypia that mimic prostate adenocarcinoma. Immunohistochemical stains for basal cell markers are most helpful in the differential diagnosis. All of the published studies show basal cell lesions are positive for basal cell keratins, whereas adenocarcinoma is negative for both. We reported two cases of prostate basal cell lesions with negative basal cell keratin expression by immunohistochemistry.

Study Design: We reported the histologic and immunohistochemical profiles of two cases of basal cell lesions of the prostate.

Results: Histologically, both cases were highly suspicious for prostate adenocarcinoma with infiltrative growth pattern and significant nuclear atypia. The atypical glands in both cases were negative for basal cell keratins. However, both lesions were positive for another basal cell marker, p63, confirming that they were basal cells in origin, rather than prostate adenocarcinoma.

Conclusion: Prostate basal cell lesions can occasionally be negative for basal cell keratins by immunohistochemistry and therefore may be misdiagnosed as prostate adenocarcinoma. We recommend using both p63 and basal cell keratins simultaneously in the workup of atypical prostate lesions to avoid such a misdiagnosis.

\section{Editorial Comment}

Absence of basal cells is a hallmark for the diagnosis of prostatic adenocarcinoma. Basal cells in the prostate do not have phenotype of myoepithelial cells and this is opposite to what happens in the mammary gland. In the latter, the myoepithelial cells have function of contraction that does not happen in the prostate. The basal cells in the prostatic gland can be recognized on hematoxylin and eosin stain. They are located next to the basement 
membrane, the nuclei may be round, oval or pyramidal, darker than the apical cells and sometimes show a clear halo. They stain in immunohistochemistry by high-weight cytokeratins (34ßE12) and p63. The first stains the cytoplasm and the latter the nuclei.

Presence of basal cells in prostatic acini excludes adenocarcinoma but not their absence. Why does it happen? One of the reasons is disclosed by the paper of this survey. Very rarely basal cells may not stain by immunohistochemistry. Most frequently, absence of basal cells is due to the anatomical distribution in the acini. Basal cells may be continuously distributed along the acini or may be patchy or discontinuously distributed. The latter distribution frequently happens in smaller branches of the acini, which may also not show basal cells at all.

This peculiar distribution of basal cells is of utmost importance for the proper interpretation of small foci "suspicious but not diagnostic for adenocarcinoma" (improperly called ASAP). In such small foci, basal cells may be absent due to anatomical spacing and not due to absence of true neoplastic acini.

Dr. Athanase Billis

Full-Professor of Pathology

State University of Campinas, Unicamp

Campinas, Sao Paulo, Brazil

\section{INVESTIGATIVE UROLOGY}

\section{Expression of cAMP and cGMP-Phosphodiesterase Isoenzymes 3, 4, and 5 in the Human Clitoris: Immunohistochemical and Molecular Biology Study}

Oelke M, Hedlund P, Albrecht K, Ellinghaus P, Stief CG, Jonas U, Andersson KE, Uckert S

Department of Urology, University of Amsterdam, Academic Medical Center, Amsterdam, The Netherlands

Urology. 2006; 67: 1111-6

Objectives: Only a little research has focused on the evaluation of female sexual function. With sexual stimulation, the clitoris becomes engorged with blood and tumescent. Nevertheless, only little is known about the significance of the cyclic nucleotide-mediated signal transduction in the control of this process. We sought to elucidate the presence of the phosphodiesterase (PDE) isoenzymes 3, 4, and 5 in the human clitoris using immunohistochemical and molecular biology methods.

Methods: Thin sections of clitoral specimens were incubated with primary antibodies directed against PDE isoenzymes 3, 4, and 5. Next, the sections were incubated with either Texas red or fluorescein isothiocyanatelabeled secondary antibodies, and visualization was done using laser microscopy. The expression of mRNA encoding for various PDE isoenzymes was evaluated using reverse transcriptase polymerase chain reaction.

Results: Immunofluorescence indicating the presence of PDE4 (cyclic adenosine monophosphate-PDE) was observed in the nonvascular smooth musculature of the corpus cavernosum clitoris, sinusoidal endothelial and subendothelial layers, and nerve fibers innervating the tissue. Immunoreactivity specific for PDE5 (cyclic guanosine monophosphate-PDE) was limited to the smooth muscle of the clitoral erectile tissue. The fluorescein isothiocyanate reaction indicating the expression of PDE3 (cyclic adenosine monophosphate-PDE) was registered to a certain degree only in the clitoral epidermis. In the reverse transcriptase polymerase chain reaction studies, a predominant expression of mRNA encoding for PDE1A was registered, but only small amounts of mRNA encoding for PDE4 and PDE5 were detected. 